RIKEN-AF-NP-295

\title{
Nonperturbative QCD corrections to the effective coefficients of the four-Fermi operators
}

\author{
Mohammad R. Ahmady ${ }^{a *}$ and Victor Elias $^{b \dagger}$ \\ ${ }^{a}$ LINAC Laboratory, The Institute of Physical and Chemical Research (RIKEN) \\ 2-1 Hirosawa, Wako, Saitama 351-0106, Japan \\ ${ }^{b}$ Department of Applied Mathematics, University of Western Ontario \\ London, Ontario, Canada N6A 5B7
}

(January 1999)

\begin{abstract}
We calculate the leading nonperturbative contributions to the effective Wilson coefficient of the four-fermion operators arising from the QCD penguin, and we demonstrate how the usual perturbative one loop contribution is augmented by nonperturbative condensates. These corrections, which are obtained by quark and gluon condensate insertions into the quark loops, enter at the next-to-leading logarithm precision. Our results indicate for the charmed quark loop that the gluon condensate contribution is quite sensitive to the momentum transfer to the quark-antiquark pair.
\end{abstract}

*Email: ahmady@riken.go.jp

†Email:zohar@apmaths.uwo.ca 
Nonleptonic B decays to light hadrons have recently received a lot of theoretical attention. This is mostly due to the measurements by the CLEO Collaboration of a number of $B \rightarrow$ $h_{1} h_{2}$ decays, where $h_{1}$ and $h_{2}$ are light hadrons such as $\pi, K, \eta^{\prime}$ and $\omega$ [1] [3]. For example, the larger than expected experimental results for the branching ratio of the inclusive $B \rightarrow X_{s} \eta^{\prime}$ and its exclusive counterpart $B \rightarrow K \eta^{\prime}$ have led to various theoretical explanations both within and beyond the Standard Model 4 8]. On the other hand, these decays could provide the first evidence for $\mathrm{CP}$ violation in $\mathrm{B}$ decays in the near future [9].

The standard theoretical framework to study the nonleptonic $B$ decays is based on the effective Hamiltonian approach. In this way, the heavy degrees of freedom, i.e. top quark and $W^{ \pm}$gauge bosons, are integrated out and the matrix element can be written as

$$
<h_{1} h_{2}\left|H_{\text {eff }}\right| B>=\frac{G_{F}}{\sqrt{2}} \sum_{i} V_{\mathrm{CKM}}^{i} C_{i}(\mu)<h_{1} h_{2}\left|O_{i}(\mu)\right| B>
$$

where $\left\{O_{i}\right\}$ is the complete set of operators relevant to $B \rightarrow h_{1} h_{2}$ decay and $C_{i}(\mu)$ are the corresponding Wilson coefficients evaluated at the renormalization scale $\mu$ [10]. These coefficients have been calculated up to the next-to-leading logarithm (NLL) precision in the strong coupling constant $\alpha_{s}$ [11, 12]. At this precision, the matrix elements on the RHS of that equation should be evaluated at the one loop level in order to have a renormalizationscheme independent result for the LHS of the eq. (1) 11, 13.

In this work, we concentrate on the four-Fermi operator subset of $\left\{O_{i}\right\}$, i.e. $i=1 . .6$, which is relevant for the hadronic $B$ decays. The first two operators $O_{1}$ and $O_{2}$ are the current-current opertors:

$$
O_{1}=\bar{q}_{\alpha} \gamma^{\mu} L u_{\alpha} \bar{u}_{\beta} \gamma_{\mu} L b_{\beta}, O_{2}=\bar{q}_{\alpha} \gamma^{\mu} L u_{\beta} \bar{u}_{\beta} \gamma_{\mu} L b_{\alpha}
$$

and the other four are the so-called QCD penguin operators:

$$
\begin{aligned}
& O_{3}=\bar{q}_{\alpha} \gamma^{\mu} L b_{\alpha} \sum_{q^{\prime}} \bar{q}_{\beta}^{\prime} \gamma_{\mu} L q_{\beta}^{\prime}, O_{4}=\bar{q}_{\alpha} \gamma^{\mu} L b_{\beta} \sum_{q^{\prime}} \bar{q}_{\beta}^{\prime} \gamma_{\mu} L q_{\alpha}^{\prime} \\
& O_{5}=\bar{q}_{\alpha} \gamma^{\mu} L b_{\alpha} \sum_{q^{\prime}} \bar{q}_{\beta}^{\prime} \gamma_{\mu} R q_{\beta}^{\prime}, O_{6}=\bar{q}_{\alpha} \gamma^{\mu} L b_{\beta} \sum_{q^{\prime}} \bar{q}_{\beta}^{\prime} \gamma_{\mu} R q_{\alpha}^{\prime} .
\end{aligned}
$$

where $q=s$ or $q=d$ for $b \rightarrow s$ or $b \rightarrow d$ transitions, respectively, and where $L$ and $R$ are the left and right handed projection operators, i.e. $L=1-\gamma_{5}$ and $R=1+\gamma_{5}$. Two more 
current-current operators which are obtained by replacing $u$ quark by $c$ quark in eq. should also be added to the above list.

In NLL precision, the matrix element of the operators is to be evaluated at one loop level, as mentioned above. These one loop matrix elements in turn can be written in terms of tree level matrix elements with loop effects included in the corresponding effective Wilson coefficients $C_{i}^{\text {eff }} 11,13$. There are two types of relevant one loop diagrams, the vertex corrections, where a gluon connects two of the outgoing quark lines, and penguin diagrams where a quark-antiquark pair forms a loop and emits a gluon which in turn produces a quark-antiquark pair. Our focus in this paper is on the latter type of corrections (Fig. 1) since they could have a potentially significant effect on branching ratios and CP asymmetry in two body hadronic $B$ decays [13,14. In fact, the absorptive portion of penguin diagrams can provide the strong phase necessary for direct CP violation. This one loop correction is analogous to the so-called continuum contribution in the dileptonic rare $B$ decays $B \rightarrow X_{s}\left(X_{d}\right) \ell^{+} \ell^{-}$, where one has to deal with a lepton-antilepton pair, as opposed to a quark-antiquark pair [15]. For the dilepton case, we also have significant nonperturbative contributions from intermediate vector mesons which are quite sensitive to the momentumtransfer to the dileptons. Motivated by the above analogy, we embark on supplementing $C_{i}^{\text {eff }}$ with a corresponding set of nonperturbative contributions.

In this work, we calculate the leading nonperturbative corrections to the perturbative evaluation of the penguin diagrams. Ignoring the vertex corrections, only the QCD penguin operators receive corrections from one loop diagrams of Fig. 1 [13]:

$$
\begin{aligned}
& C_{3}^{\mathrm{eff}}=C_{3}-\frac{1}{6} \frac{\alpha_{s}}{4 \pi}\left(C_{t}+C_{p}\right)+\ldots, \\
& C_{4}^{\mathrm{eff}}=C_{4}+\frac{1}{2} \frac{\alpha_{s}}{4 \pi}\left(C_{t}+C_{p}\right)+\ldots, \\
& C_{5}^{\mathrm{eff}}=C_{5}-\frac{1}{6} \frac{\alpha_{s}}{4 \pi}\left(C_{t}+C_{p}\right)+\ldots, \\
& C_{6}^{\mathrm{eff}}=C_{6}+\frac{1}{2} \frac{\alpha_{s}}{4 \pi}\left(C_{t}+C_{p}\right)+\ldots .
\end{aligned}
$$

$C_{t}$ and $C_{p}$ are the loop corrections corresponding to the current-current (tree) and penguin type four-fermion operator insertion in Figure 1, respectively. Using naive dimensional 
regularization(NDR) along with the $\overline{\mathrm{MS}}$ renormalization scheme, one obtains

$$
\begin{aligned}
C_{t}= & -\sum_{q^{\prime}=u, c} \frac{V_{q^{\prime} b} V_{q^{\prime} q}^{*}}{V_{t b} V_{t q}^{*}}\left[\frac{2}{3}+\frac{2}{3} \operatorname{Ln}\left(\frac{m_{q^{\prime}}^{2}}{\mu^{2}}\right)-\Delta F_{1}\left(\frac{k^{2}}{m_{q^{\prime}}^{2}}\right)\right] C_{1} \\
C_{p}= & {\left[\frac{4}{3}+\frac{2}{3} \operatorname{Ln}\left(\frac{m_{s}^{2}}{\mu^{2}}\right)+\frac{2}{3} \operatorname{Ln}\left(\frac{m_{b}^{2}}{\mu^{2}}\right)-\Delta F_{1}\left(\frac{k^{2}}{m_{s}^{2}}\right)-\Delta F_{1}\left(\frac{k^{2}}{m_{b}^{2}}\right)\right] C_{3} } \\
& +\sum_{q^{\prime}=u, d, s, c, b}\left[\frac{2}{3} \operatorname{Ln}\left(\frac{m_{q^{\prime}}^{2}}{\mu^{2}}\right)-\Delta F_{1}\left(\frac{k^{2}}{m_{q^{\prime}}^{2}}\right)\right]\left(C_{4}+C_{6}\right)
\end{aligned}
$$

where the function $\Delta F_{1}(z)$ is defined as

$$
\Delta F_{1}(z)=-4 \int_{0}^{1} d x x(1-x) \ln [1-z x(1-x)]
$$

We note that the effective coefficients in NLL depend on the momentum-transfer $k^{2}$ as well as the internal quark mass $m_{q}$. In fact, for $z \geq 4, \Delta F_{1}(z)$ develops an imaginary part which has been taken as the strong phase necessary for generating direct CP asymmetry [13]. Note that the light internal quarks produce the dominant contributions; consequently, the larger factor $C_{t}\left(C_{1}\right.$ is much larger then $\left.C_{3 \ldots 6}\right)$ is more significant for $b \rightarrow d$ transitions in which the ratio of CKM factors for tree and penguin is not too small. Nevertheless, one can not have a complete picture of the NLL corrections without taking into account nonperturbative effects which, as in the case of the intermediate resonance contribution to $B \rightarrow X_{s}\left(X_{d}\right) \ell^{+} \ell^{-}$, can prove to be significant. To investigate this possibility, we calculate the leading nonperturbative contributions to $C_{i}^{\text {eff }}, i=3 . .6$ by inserting quark and gluon condensates, which are believed to be responsible for the chiral-symmetry breaking and confinement properties, respectively, of the QCD vacuum, into one-loop penguin diagrams (Figs. 2,3).

QCD condensates characterize the nonperturbative content of the QCD vacuum, and therefore, condensate insertions should give us a hint of the leading nonperturbative contributions to the effective Wilson coefficients. To demonstrate our method, we present a detailed calculation of the quark condensate result. For the gluon condensate contribution, the necessary expressions are extracted from previously determined [16] condensate contributions to the vector correlation function. 
To obtain the quark condensate contribution, we proceed by replacing the usual perturbative internal quark propagator in Fig. 1 with the full quark propagator $S(p)$ :

$$
S(p)=S^{\mathrm{P}}(p)+S^{\mathrm{NP}}(p) .
$$

The nonperturbative contributions to the quark propagator can then be written as

$$
i S^{\mathrm{NP}}(p)=\int d^{4} x e^{i p . x}<\Omega|: \Psi(x) \bar{\Psi}(0):| \Omega>,
$$

where $\mid \Omega>$ is the nonperturbative $\mathrm{QCD}$ vacuum. The nonlocal vacuum expectation value (vev) in eq. (8) can be expanded in terms of local condensates [17]. To ascertain how the leading nonperturbative QCD contributions affect the coefficients $C_{i}^{\text {eff }}$, only the lowest dimensional (quark and gluon condensates) components of the expansion are considered below. However, we note that the nonlocal vev of two quark fields does not contain a gluon condensate component. The quark condensate projection of the nonperturbative quark propagator is taken from Ref. [18]:

$$
i S^{\mathrm{NP}}(p)=(2 \pi)^{4}\left(\not p+m_{q}\right) F(p)
$$

where the Fourier transform of $F(p)$ is expressed in terms of Bessel function

$$
\int d^{4} p e^{-i p \cdot x} F(p)=-\frac{<\bar{q} q>}{6 m_{q}^{2}} \frac{J_{1}\left(m_{q} \sqrt{x^{2}}\right)}{\sqrt{x^{2}}},
$$

with the following important property:

$$
\left(p^{2}-m_{q}^{2}\right) F(p)=0
$$

The dimension-3 quark condensate $<\bar{q} q>$ is the vev of the normal ordered local product of quark and antiquark fields, i.e.

$$
<\bar{q} q>=<\Omega|: \bar{\Psi}(0) \Psi(0):| \Omega>.
$$

Using the Feynman rule of eq. (9), one can obtain the nonperturbative $<\bar{q} q>$ contribution to the effective Wilson coefficients in a straightforward manner. The relevant Feynman 
diagram is illustrated in Fig. 2, where the nonperturbative quark propagator $S^{\mathrm{NP}}$ is depicted by a disconnected line with two dots. Here we concentrate on the loop portion of Fig. 2 which differs from the perturbative case of Fig. 1. Aside from the color factor, the vector current correlation function can be written as

$$
\Pi_{\mu \nu}^{<\bar{q} q>}(k)=2 \int d^{4} p \frac{\operatorname{Tr}\left[(\not p-\not k+m) \gamma_{\mu}(\not p+m) \gamma_{\nu}\right] F(p)}{(p-k)^{2}-m^{2}+i \epsilon}
$$

where the factor 2 in front is due to two possible insertions of the nonperturbative quark propagator in the fermion loop. By contracting $p^{\mu} p^{\nu}$ into $\Pi_{\mu \nu}^{<\bar{q} q>}$ and using the identity

$$
\begin{aligned}
\int d^{4} p k \cdot p F(p) & =i \lim _{\xi \rightarrow 0} \frac{d}{d \xi} \int d^{4} p e^{-i \xi k \cdot p} F(p) \\
& =-i \frac{<\bar{q} q>}{6 m_{q}} \lim _{\xi \rightarrow 0} \frac{d}{d \xi} \frac{J_{1}\left(m_{q} \xi \sqrt{k^{2}}\right)}{m_{q} \xi \sqrt{k^{2}}}=0
\end{aligned}
$$

where the second line is derived via eq. (10), one can show explicitly the transversality of the correlation function in eq. (13). Consequently, one finds upon contracting $g^{\mu \nu}$ into $\Pi_{\mu \nu}^{<\bar{q} q>}$ and imposing the on-shell constraint (11) that

$$
\Pi_{\mu}^{<\bar{q} q>\mu}(k)=24\left(2 m_{q}^{2}+k^{2}\right) \int d^{4} p \frac{F(p)}{k^{2}-2 k \cdot p+i \epsilon}-24 \int d^{4} p F(p) .
$$

The integrals appearing in eq. (15) are evaluated as follows [18]:

$$
\begin{aligned}
\int d^{4} p F(p) & =-\frac{<\bar{q} q>}{12 m_{q}} \\
\int d^{4} p \frac{F(p)}{k^{2}-2 k \cdot p+i \epsilon} & =\frac{i<\bar{q} q>}{12 m_{q}^{2} \sqrt{k^{2}}} \int_{0}^{\infty} \frac{d \eta}{\eta} e^{i \eta k^{2}-\epsilon \eta} J_{1}\left(2 \eta m_{q} \sqrt{k^{2}}\right) \\
& =-\frac{<\bar{q} q>}{6 m_{q} k^{2}}\left[1+\sqrt{1-\frac{4 m_{q}^{2}}{k^{2}}}\right]^{-1}
\end{aligned}
$$

where the final line is derived by utilizing a tabulated integral [19]. Using the transversality of $\Pi_{\mu \nu}^{<\bar{q} q>}$, the result for the RHS of (13) is easily obtained:

$$
\Pi_{\mu \nu}^{<\bar{q} q>}(k)=-\frac{<\bar{q} q>}{3 m_{q}^{3}}\left[1-\left(1+\frac{2 m_{q}^{2}}{k^{2}}\right) \sqrt{1-\frac{4 m_{q}^{2}}{k^{2}}}\right]\left(g_{\mu \nu} k^{2}-k_{\mu} k_{\nu}\right),
$$

This result is, in fact the $<\bar{q} q>$-contribution to the vector-current correlation function derived in ref. [16]. The corresponding gluon condensate contribution to the vector-current correlator, which may be derived by several different methods [18], is given by [16] 


$$
\begin{aligned}
\Pi_{\mu \nu}^{<G^{2}>}(k) & =\left(k_{\mu} k_{\nu} / k^{2}-g_{\mu \nu}\right) \frac{\alpha_{s}<G^{2}>}{12 \pi k^{2}(1-4 / z)^{2}} \\
& \times\left\{\frac{12}{z}\left(\frac{1}{z^{2}}-\frac{2}{z^{4}}\right) X(z)-\left(1-\frac{4}{z}+\frac{12}{z^{2}}\right)\right\}
\end{aligned}
$$

where

$$
z \equiv k^{2} / m_{q}^{2}
$$

and where 16,20

$$
\begin{aligned}
X(z) \equiv & \frac{1}{(1-4 / z)}\left[\int_{0}^{1} d x \ln [1-z x(1-x)-i|\epsilon|]+2\right] \\
= & {\left[\begin{array}{ll}
\frac{1}{\sqrt{1+4 /|z|}} \ln \left[\frac{\sqrt{1+4 /|z|}+1}{\sqrt{1+4 /|z|}-1}\right], & z<0 \\
\frac{2 \sqrt{4 / z-1}}{(1-4 / z)} \tan ^{-1}\left[\frac{1}{\sqrt{4 / z-1}}\right], & 0<z<4 \\
\frac{1}{\sqrt{1-4 / z}}\left(\ln \left[\frac{1+\sqrt{1-4 / z}}{1-\sqrt{1-4 / z}}\right]-i \pi\right), & z>4
\end{array}\right] }
\end{aligned}
$$

It is evident from (18) and (20) that this contribution diverges sharply at $\mathrm{z}=4$ [21].

Consequently, we replace eq. (6) with the following $<\bar{q} q>$ - and $<G^{2}>$-augmented definition for $\Delta F_{1}$ in order to include the leading nonperturbative quark and gluon condensate contributions to the effective Wilson coefficients (eq. (4)):

$$
\begin{aligned}
\Delta F_{1}(z) & =-4 \int_{0}^{1} d x x(1-x) \ln [1-z x(1-x)] \\
& +\frac{8 \pi^{2}<m_{q} \bar{q} q>}{3 m_{q}^{4}}\left[1-\left(1+\frac{2}{z}\right) \sqrt{1-\frac{4}{z}}\right] \\
& +\frac{\pi<\alpha_{s} G^{2}>}{6 m_{q}^{4}} \frac{1}{z^{2}\left(1-\frac{4}{z}\right)^{2}}\left[\frac{48\left(1-\frac{2}{z}\right)}{z^{2}} X(z)-4+\frac{16}{z}-\frac{48}{z^{2}}\right],
\end{aligned}
$$

where the second term is written in terms of the renormalization group (RG) invariant combination $<m_{q} \bar{q} q>$, and where the factor $\alpha_{s}$ in the third term is absorbed in the gluon condensate order parameter to achieve approximate RG-invariance.

In the decays $B \rightarrow h_{1} h_{2}$, where $h_{1}$ and $h_{2}$ are light mesons, the characteristic range of $k^{2}$ is argued to be 22

$$
\frac{m_{b}^{2}}{4} \lesssim k^{2} \lesssim \frac{m_{b}^{2}}{2}
$$


For light quarks $\left(z=k^{2} / m_{q}^{2}>>1\right)$, the nonperturbative terms in eq. (21) can be approximated by

$$
\Delta F_{1}^{\text {nonperturbative }} \approx \frac{16 \pi^{2}<m_{q} \bar{q} q>}{k^{4}}-\frac{2 \pi<\alpha_{s} G^{2}>}{3 k^{4}}
$$

Inserting the phenomenological values $<\bar{q} q \approx(-0.25)^{3} \mathrm{GeV}^{3}$ and $<\alpha G^{2}>\approx 0.045$ $\mathrm{GeV}^{4}$, we observe that the leading nonperturbative corrections are around three orders of magnitude smaller than the perturbative contribution, and can therefore be safely neglected.

For the heavier charmed quark, however, the situation is more interesting because of the presence of a singularity at $z=4$ in the gluon-condensate term: the range $4 G e V^{2} \lesssim k^{2} \lesssim$ $10 \mathrm{GeV}^{2}$ suggested by (22) is quite likely inclusive of $z=4$, (i.e., of $k^{2}=4 m_{c}^{2}$ ) given the present 1.1-1.4 GeV range of values for $m_{c}$ [23]. Consequently, there is a genuine possibility that the gluon-condensate contribution to the penguin amplitude could be comparable to the purely perturbative correction, depending on which value of $k^{2}$ one chooses. This sensitivity to the momentum transfer $k^{2}$ is also important for investigating direct CP asymmetry in hadronic B decays, as the gluon-condensate contribution to (21) develops an imaginary part which dominates the strong phase of the effective Wilson coefficients as $z \rightarrow 4$ from above.

For a physical interpretation of the above results, we again use the analogy with the dileptonic $\mathrm{B}$ decays $B \rightarrow X_{s} \ell^{+} \ell^{-}$. These decay modes receive a perturbative charmed-quark loop correction (continuum contribution) as well as nonperturbative contributions when $c \bar{c}$ forms intermediate resonances such as $J / \psi, \psi^{\prime}, \ldots$ [15]. The latter can be incorporated by modelling a Breit-Wigner form for the resonance propagator in the long distance amplitude 24

$$
A^{\text {nonperturbative }} \sim \frac{1}{M^{2}-k^{2}-i M \Gamma}
$$

where $k$ is the momentum transfer to dileptons, and where $M$ and $\Gamma$ are the mass and total decay width of the intermediate resonance. The resulting dileptonic differential decay rate has peaks at $k^{2} \sim M^{2}$, and the nonperturbative resonance contribution indeed dominates the branching ratio of this mode. 
One can make a similar charmonium-resonance interpretation for the singularity at $k^{2}=$ $4 m_{c}^{2}$ in the $<G^{2}>$ contribution to the effective Wilson coefficients of the condensateaugmented penguin amplitude, particularily if we identify the $k^{2}>4 m_{c}^{2}$ kinematic threshold for the imaginary part with the onset of a "physical" intermediate state. It must be noted, however, that such a resonance couples directly to a single gluon (Fig. 3), necessarily implying that the $z=4$ singularity, if indeed a resonance, must be associated with the leading contribution of a weakly bound colour-octet c $\bar{c}$ state [25].

In conclusion, we have calculated the nonperturbative quark and gluon condensate contributions to the effective Wilson coefficients of the QCD penguin four-fermion operators, supplementing the one loop (continuum) $q \bar{q}$ correction which appears at NLL precision with its nonperturbative counterparts. We have pointed out how incorporation of the gluon condensate in the charmed quark loop results in a contribution which is highly sensitive to momentum transfer $k^{2}$. Since $k^{2}$ is not in-and-of itself a physical observable in two body hadronic B decays, one cannot exclude the possibility of significant nonperturbative contributions to both the magnitude and the strong phase of the effective Wilson coefficients of the four-Fermi operators $\mathrm{O}_{3} \ldots O_{6}$.

\section{ACKNOWLEDGEMENTS}

MRA would like to thank Emi Kou for useful discussions and comments on quark condensate contribution. VE is grateful for support from the Natural Sciences and Engineering Research Council of Canada. MRA acknowledges support from the Science and Technology Agency of Japan. 


\section{REFERENCES}

[1] J. G. Smith, preprint COLO-HEP-395 (1998), hep-ex/9803028.

[2] B. H. Behrens et al. (CLEO Collaboration), Phys. Rev. Lett. 80, 3710 (1998).

[3] T. E. Browder et al. (CLEO Collaboration), Phys. Rev. Lett. 81, 1786 (1998).

[4] D. Atwood and A. Soni, Phys. Lett. B405, 150 (1997).

[5] W.-S. Hou and B. Tseng, Phys. Rev. Lett. 80, 434 (1998).

[6] I. Halperin and A. Zhitnitsky, Phys. Rev. D56, 7247 (1997); Phys. Rev. Lett. 80, 438 (1998).

[7] F. Yuan and K. T. Chao, Phys. Rev. D56, 2495 (1997).

[8] M. R. Ahmady, E. Kou and A. Sugamoto, Phys. Rev. D58, 014015 (1998).

[9] M. R. Ahmady and E. Kou, hep-ph/9807398, to appear in Phys. Rev. D.

[10] For a recent detailed review see A. J. Buras, hep-ph/9806471.

[11] A. J. Buras, M. Jamin, E. Lautenbacher and P. H. Weisz, Nucl. Phys. B370, 69 (1992);

[12] M. Ciuchini, E. Franco, G. Martinelli and L. Reina, Nucl. Phys. B415, 403 (1994).

[13] G. Kramer, W. F. Palmer and H. Simma, Nucl. Phys. B428, 77 (1994); Z. Phys. C66, $429(1995)$.

[14] A. Ali, G. Kramer and Cai-Dian Lü, Phys. Rev. D58, 094009 (1998); Phys. Rev. D59, 014005 (1999).

[15] M. R. Ahmady, Phys. Rev. D53, 2843 (1996).

[16] E. Bagan, J. I. Latorre and P. Pascual, Z. Phys. C32, 43 (1986).

[17] V. Elias, T. G. Steele and M. D. Scadron, Phys. Rev. D38, 1584 (1988). 
[18] E. Bagan, M. R. Ahmady, V. Elias and T. G. Steele, Z. Phys. C61 157 (1994); Phys. Lett. B305, 151 (1993).

[19] I. S. Gradshteyn and I. M. Ryzhik, Table of Integrals, Series and Products (Academic Press, Orlando, 1980) p 712, Eq (6.623.3).

[20] A. S. Deakin et al, Eur. Phys. J. C4, 693 (1998).

[21] This divergence is not removed by the operator realignment suggested by M. Jamin and M. Münz, Z. Phys. C60, 569(1993). Such a change of basis would add a linear combination of $<m_{q} \bar{q} q>$ - and $<\bar{q} \sigma \cdot G q>$-coefficients to the $<\alpha_{s} G^{2}>$-coefficient extracted from (18). At $\mathrm{z}=4$, the $<m_{q} \bar{q} q>$-coefficient is finite, and the mixed-condensate $<\bar{q} \sigma \cdot G q>$-coefficient [16] is only softly divergent $\left(C_{M} \sim-\{1-4 / z\}^{-1 / 2} / 6 m^{3}\right)$.

[22] H. Simma and D. Wyler, Phys. Lett. B272, 395 (1991); N. G. Deshpande and J. Trampetic, Phys. Rev. D41, 2926 (1990)

[23] C. Caso et al [Particle Data Group], Eur. Phys. J., C3, 1 (1998).

[24] C. S. Lim, T. Morozumi and A. I. Sanda, Phys. Lett. B218, 343 (1989); N. G. Deshpande, J. Trampetic and K. Penose, Phys. Rev. D39, 1461 (1989).

[25] Confinement of such a state presumably entails additional dynamics. The idea of colournonsinglet intermediate states having some impact at colour-singlet asymptopia is hardly a new one, though such states are more typically indentified with quarks and gluons. 


\section{Figure Captions}

Figure 1: Perturbative one loop correction to the effective coefficient of the four-fermion operators.

Figure 2: Nonperturbative quark condensate contribution to the effective coefficient of the four-fermion operators at the one-loop level.

Figure 3: Nonperturbative gluon condensate contribution to the effective coefficient of the four-fermion operators at the one-loop level. 


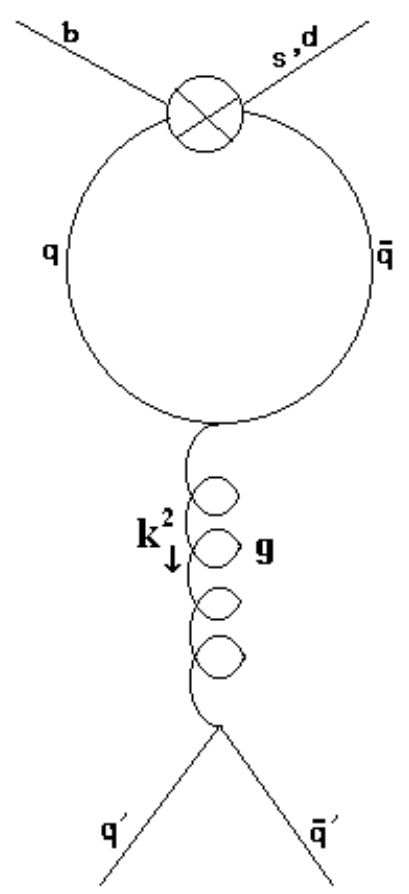

Figure 1 


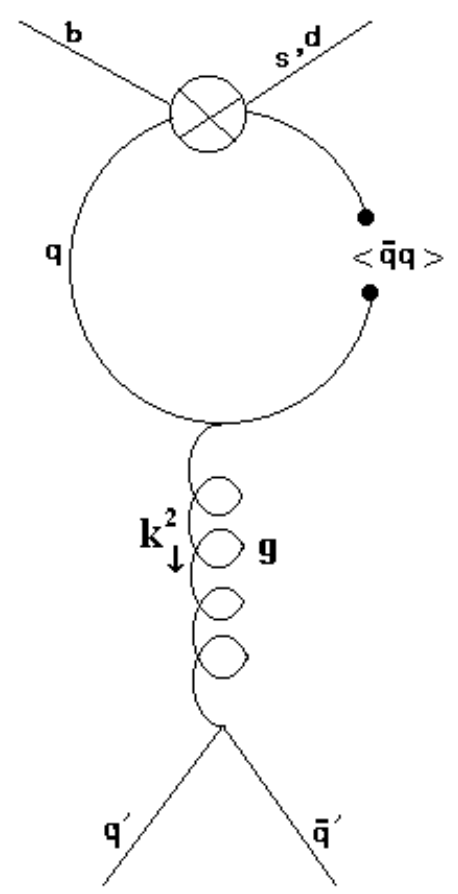

Figure 2 

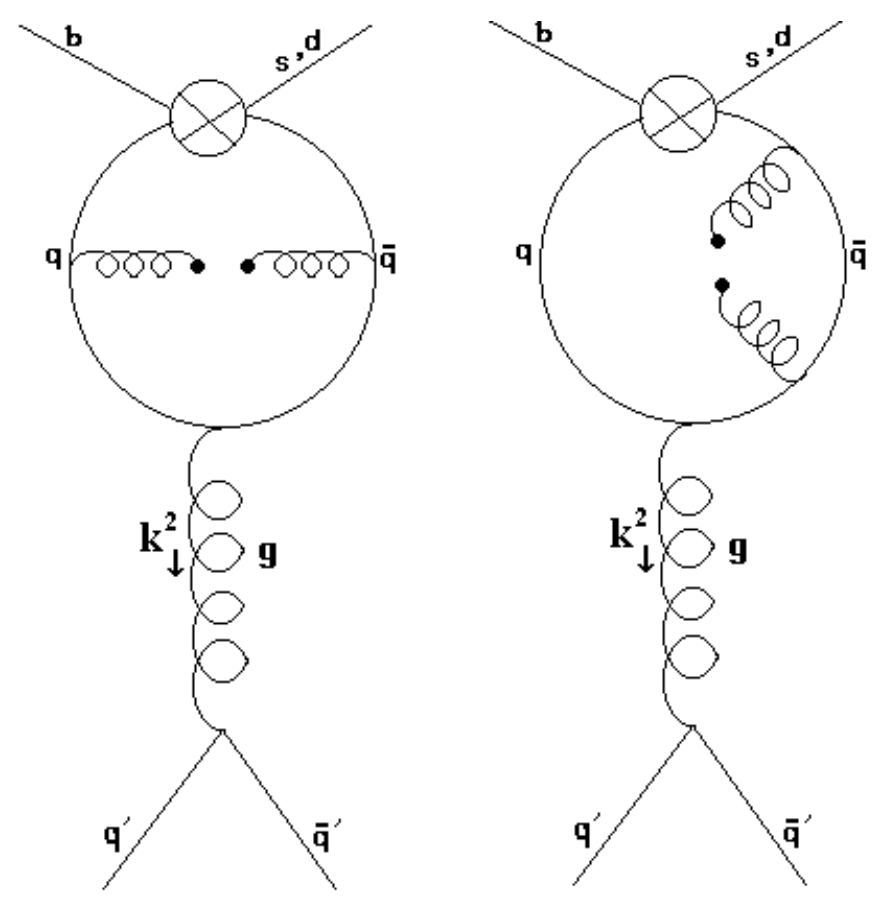

Figure 3 